\title{
The predictive value of the prognostic nutritional index for contrast-induced nephropathy
}

Murat Gucun ${ }^{1}$, Serkan Senger², Muzaffer Kahyaoğluª ${ }^{3}$ Okan Akyuz', Mehmet Celik ${ }^{4}$, Ahmet Guner ${ }^{5}$, Yusuf Yilmaz $^{6}$

${ }^{1}$ Department of Nephrology, Kartal Kosuyolu Training and Research Hospital, Istanbul, Turkey

${ }^{2}$ Department of General Surgery, Kartal Kosuyolu Training and Research Hospital, Istanbul, Turkey

${ }^{3}$ Department of Cardiology, Beykoz State Hospital, Istanbul, Turkey

${ }^{4}$ Department of Cardiology, Kartal Kosuyolu Training and Research Hospital, Istanbul, Turkey

${ }^{5}$ Department of Cardiology, Mehmet Akif Ersoy Thoracic and Cardiovascular Surgery Center, Training and Research Hospital, Istanbul, Turkey

${ }^{6}$ Department of Cardiology, Goztepe Training and Research Hospital, Istanbul, Turkey

Received: 2021-09-01.

Accepted: 2022-01-12

This work is licensed under a

Creative Commons Attribution 4.0 International License

J Clin Med Kaz 2022; 19(1):46-49

Corresponding author:

Muzaffer Kahyaoğlu.

E-mail: mkahyaoglu88@gmail.com;

ORCID: 0000-0002-8572-1725

\begin{abstract}
Aim: This study aims to evaluate the association between prognostic nutritional index (PNI) and contrast-induced nephropathy (CIN).

Material and methods: A total of 251 patients who were at high risk for contrast nephropathy were included in the study. The patients were grouped according to their PNI score (PNI score $<45$ or PNI score 245). CIN was defined as a $25 \%$ relative increase, or $0.5 \mathrm{mg} / \mathrm{dL}$ absolute increase in serum creatinine level above baseline within 72 hours of contrast exposure, in the absence of an alternative explanation.

Results: Two groups were assigned according to the PNI score. The first group consists of 111 patients $(\mathrm{PNI}<45)$ and the second group has 140 patients (PNI $\geq 45)$. CIN developed in 162 (\%64.8) patients. C-reactive protein was higher in the low-PNI group. Also, the patients with the lowPNI group had lower ejection fraction, lower serum albumin levels, and lower hemoglobin levels. CIN, postprocedure renal replacement therapy requirement and in-hospital mortality were higher in the low PNI group. Multivariable logistic regression analysis revealed that advanced age $(p=0.012,[O R]=1.044$ [1.009-1.079]), low baseline GFR $(p=0.033,[O R]=$ 1.022 [1.002-1.043]), high amount of contrast media $(p=0.022,[O R]=1.017$ [1.002-1.031]), and low PNI score ( $p=0.033,[O R]=2.069$ [1.060-4.039]) were independent predictors of CIN.
\end{abstract}

Conclusion: Our study demonstrated that the PNI score was an independent risk factor for the development of CIN.

Key words: prognostic nutritional index, contrast induced nephropathy, coronary angiography, percutaneous coronary intervention

\section{Introduction}

Contrast-induced nephropathy (CIN) is a potentially benign form of acute kidney injury (AKI) that occurs following contrast media administration. In most cases, the glomerular filtration rate (GFR) mildly decreases within three to seven days and then returns to baseline or close to baseline values. CIN is a common cause of AKI in hospitalized patients, and the incidence of it varies from $3 \%$ to $30 \%$ in different studies due to the contrast volume, accompanying risk factors, and route of administration [1]. Although it is generally reversible, CIN development is associated with increased prolonged hospitalization and mortality [2]. Once it occurs, there are no spesific treatment options for CIN yet [3]. Therefore, it is very important to identify the population at risk and take measures to prevent AKI progression [3].

Prognostic nutritional index (PNI) has been introduced as a simple indicator of nutritional and inflammatory status in cancer patients $[4,5]$. It is calculated by using serum albumin concentration and total lymphocyte count. PNI has also been proposed to indicate the prognosis of heart failure and ST-segment elevated myocardial infarction (STEMI) $[6,7]$. The aim of this study is to explore the predictive effect of PNI on CIN in patients that underwent coronary angiography (CAG) or percutaneous coronary intervention (PCI). 


\section{Materials and methods}

Between October 2016 and March 2018, patients requiring nephrology consultation before CAG or PCI because of the high risk for CIN were included in this study. The exclusion criteria were; 1) exposure to contrast media less than one week before the procedure, 2) exposure to nephrotoxic drug less than ten days before the procedure, 3 ) severe hepatic disease, 4) active malignancy, 5) active infectious disease, 6) recent major surgical procedure or trauma, 7) being presented with other causes of AKI, 8) being treated with hemodialysis.

A total of 69 patients were excluded from the study. Fifty patients were excluded because of having at least one exclusion criterion and 19 patients for missing data on serum albumin or total lymphocyte count. Finally, 251 patients were eligible for the study. The study flow chart was demonstrated in Figure 1. PNI was calculated with the formula: $10 \mathrm{x}$ serum albumin $(\mathrm{g} / \mathrm{dL})$ $+0.005 \mathrm{x}$ total lymphocyte count (per mm3). The PNI cut-off score of 45 points has been used in previous studies and has been confirmed as optimal $[6,8]$. Based on those results, we chose a score of 45 points as an optimal cut-off value. Two groups were assigned according to the PNI score. The first group was 111 patients $(\mathrm{PNI}<45)$ and the second group was 140 patients (PNI $\geq 45)$.

Figure 1 - Study flow-chart

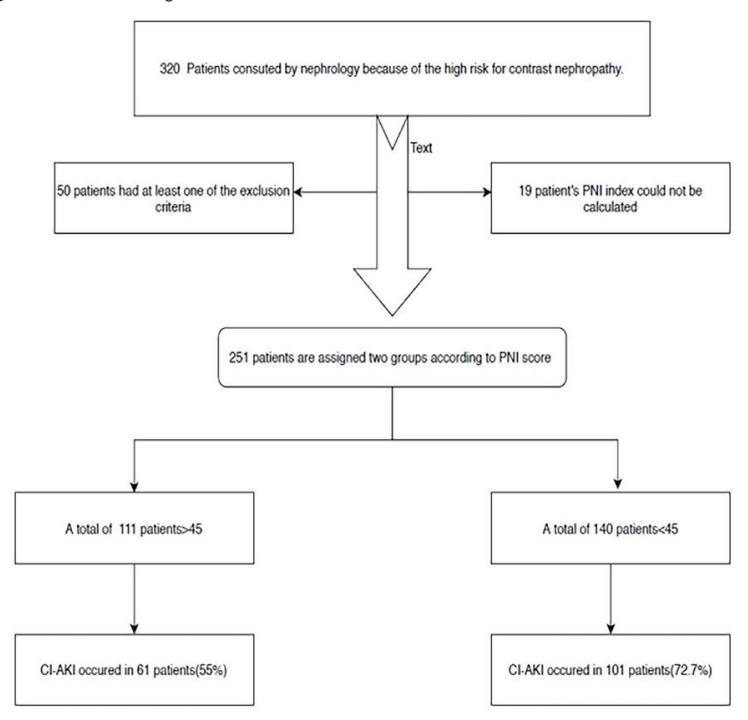

Samples for hemogram and biochemical parameters were taken from the patients at the time of admission and the serum creatinine $(\mathrm{SCr})$ level was measured daily postprocedure until the discharge. All enrolled cases were received hydration $(0.9 \%$ normal saline at $0.5-1.0 \mathrm{~mL} / \mathrm{kg} / \mathrm{h}$ ) for $3-12 \mathrm{~h}$ before CAG or $\mathrm{PCI}$ and it was continued for up to $12 \mathrm{~h}$ after CAG depending on the patient's volume status. N-acetylcysteine $(>1200 \mathrm{mg} /$ day) treatment was started 1 day before CAG or PCI and ceased up 2 days after the procedure. The contrast medium (iohexol) used for the procedure was nonionic, iso-osmolar. Within the 72 hours following intravascular contrast media administration; an absolute rise of $0.5 \mathrm{mg} / \mathrm{dL}(44 \mu \mathrm{mol} / \mathrm{L})$, or a relative $25 \%$ rise from the baseline, in serum creatinine value, in the absence of other causes was defined as CIN [9]. The Chronic Kidney Disease Epidemiology Collaboration (CKD-EPI) equation was used for GFR calculation [10].

\section{Statistical analysis}

Descriptive statistics were presented as percentage of the total $(\%)$ for categorical variables and mean \pm standard deviation for continuous variables. Mann-Whitney U test or Student's t-test was used for comparing the differences in continuous variables between the two groups. The KolmogorovSmirnov test was used to evaluate the distribution of continuous variables. Categorical variables compared with the Chi-square test or Fisher's exact test. Binary logistic regression analysis was employed to determine the independent predictors of CIN. Data were analyzed using the Statistical Package for Social Sciences Software (SPSS 17.0 Chicago, Illinois). The statistical significance was considered when a $p$-value $\leq 0.05$.

\section{Results}

A total of 251 high-risk patients were enrolled in the present study (mean age $68.4 \pm 9,8,68.10 \%$ male). The basal creatinine level was $1.76(0.63-4.17) \mathrm{mg} / \mathrm{dl}$, hemoglobin level was $11.6(7.6-18.3) \mathrm{g} / \mathrm{dl}$, ejection fraction was $50 \%(20 \%$ $65 \%$ ), C-reactive protein (CRP) value was $2,86 \pm 3.85 \mathrm{mg} / \mathrm{dl}$. The amount of contrast media used during the procedure was $52.75 \pm 28.63 \mathrm{ml}$, and CIN developed in 162 (64.8\%) patients. Baseline demographic and clinical characteristics of the patients categorized according to PNI value is given in Table 1.

Gender, history of diabetes mellitus, and pre-procedure $\mathrm{SCr}$ levels were similar in both groups. The values of CRP were higher in the low-PNI group. Whereas the patients with the lowPNI group had lower ejection fraction, lower serum albumin levels, and lower hemoglobin levels. The occurrence of CIN, post procedure renal replacement therapy requirement and inhospital mortality were higher in the low PNI group.

The variables that are thought to be associated with CIN were conducted in a univariable model to investigate risk factors for CIN. PNI ( $p=0.004$, odds ratio[OR] $=2.179$ [95\% CI: $[1.285-$ $3.694])$, female gender $(p=0.002$, $[\mathrm{OR}]=2.5[1.385-4.513])$, diabetes mellitus $(\mathrm{p}=0.007,[\mathrm{OR}]=2.019$ [1.210-3.366]), age $(\mathrm{p}$ $=0.018,[\mathrm{OR}]=1.032[1.006-1.059])$, amount of contrast media $(p=0.023,[\mathrm{OR}]=1.012[1.002-1.023])$, and hemoglobin levels $(\mathrm{p}<0.001,[\mathrm{OR}]=0.768[0.672-0.878])$ were associated with the development of CIN. In multivariable logistic regression analysis, advanced age $(\mathrm{p}=0.012,[\mathrm{OR}]=1.044$ [1.009-1.079]), low baseline GFR ( $\mathrm{p}=0.033,[\mathrm{OR}]=1.022$ [1.002-1.043]), high amount of contrast media $(\mathrm{p}=0.022,[\mathrm{OR}]=1.017[1.002-$ $1.031])$, and low PNI score $(\mathrm{p}=0.033,[\mathrm{OR}]=2.069[1.060-$ $4.039]$ ) were found as independent predictors of CIN (Table 2).

\section{Discussion}

With this study, the association between PNI and CIN following CAG or PCI was evaluated, and it is shown that PIN was a strong predictor of CIN. Besides, PNI was associated with an increased rate of mortality, and renal replacement therapy requirements.

PNI is a unified nutritional-inflammatory score, based on serum albumin levels and the lymphocyte count, reflecting the immunological and nutritional status of patients. Initially, it had been used to indicate postoperative outcomes of malnourished cancer patients undergoing gastrointestinal surgery [11]. Subsequently, it has been stated that PNI was a simple and useful predictor of prognosis in end-stage liver disease, heart failure, and STEMI $[6,7,12]$.

The pathogenesis of CIN is not entirely understood. However, hemodynamic injury, systemic inflammation, and toxic injury are accused mechanisms for the development of CIN. Especially, multiple inflammation-related factors, such as endothelial dysfunction, oxidative stress, and renal vasoconstriction are thought to influence the development of 


\begin{tabular}{|c|c|c|c|}
\hline & $\mathrm{PNI}<45$ & $\mathrm{PNI} \geq 45$ & P value \\
\hline Age & $69.6 \pm 9.3$ & $66.9 \pm 10.3$ & 0.030 \\
\hline Gender (Female) & $46(32.9 \%)$ & $34(30.6 \%)$ & 0.707 \\
\hline Diabetes Mellitus(\%) & $88(62.9 \%)$ & $59(\% 53.2)$ & 0.121 \\
\hline Ejection Fraction(\%) & $47,5(20-60)$ & $50(20-65)$ & 0.045 \\
\hline Preprocedure $\operatorname{Cr}(\mathrm{mg} / \mathrm{dl})$ & $1.845(0.63-4,17)$ & $1.6(0.65-3.5)$ & 0.321 \\
\hline $\begin{array}{l}\text { Preprocedure } \\
\text { e-GFR }\left(\mathrm{ml} / \mathrm{min} / 1.73 \mathrm{~m}^{2}\right)\end{array}$ & $38(3.37-108)$ & $40.4(14-147)$ & 0.049 \\
\hline CRP(mg/L) & $1.38(0,34-23.2)$ & $0.79(0.2-17.5)$ & 0.002 \\
\hline $\operatorname{Albumin}(\mathrm{g} / \mathrm{L})$ & $3.29(2.18-4.10)$ & $3.97(2.57-5.4)$ & $<0.001$ \\
\hline $\mathrm{WBC}\left(10^{3} / \mathrm{ml}\right)$ & $8.5(1,7-24)$ & $8.5(3.9-20)$ & 0.671 \\
\hline $\operatorname{Hemoglobin}(g / d l)$ & $10.9(7.7-18.3)$ & $12(7.6-16.9)$ & $<0.001$ \\
\hline LDL cholesterol(mg/dl) & $120.6 \pm 41.3$ & $130.7 \pm 44.3$ & 0.136 \\
\hline Triglycerides(mg/dl) & $191 \pm 91$ & $157.7 \pm 88.6$ & 0.019 \\
\hline \multicolumn{4}{|l|}{ Postprocedure } \\
\hline $\mathrm{e}-\mathrm{GFR}\left(\mathrm{ml} / \mathrm{min} / 1.73 \mathrm{~m}^{2}\right)$ & $22.75(8.0-70.7)$ & $30(6-154)$ & 0.002 \\
\hline CI-AKI(\%) & $101(72.8 \%)$ & $61(55 \%)$ & 0.04 \\
\hline RRT (\%) & $37(26.40 \%)$ & $10(9 \%)$ & $<0.001$ \\
\hline İn-hospital mortality(\%) & $13(9.3 \%)$ & $2(1.8 \%)$ & 0.013 \\
\hline
\end{tabular}

Abbreviations: Cr-creatinine; e-GFR-estimated glomerular filtration rate; CRP-C-reaktive protein; WBC-white blood cell; CI-AKI-contrast induced acute kidney injury; RRT-renal replacement theraphy

\begin{tabular}{lllll} 
& \multicolumn{1}{l}{ Univariable } & & Multivariable & \\
\cline { 2 - 6 } & OR(\%95 CI of OR) & p value & OR(\%95 CI of OR) & p value \\
PNI & $2.179[1.285-3.694]$ & 0.004 & $2.069[1.060-4.039]$ & 0.033 \\
Gender(female) & $2.5[1.385-4.513]$ & 0.002 & $2.087[0.977-4.457]$ & 0.057 \\
Age(years) & $1.032[1.006-1.059]$ & 0.018 & $1.044[1.009-1.079]$ & 0.012 \\
DM & $2.019[1.210-3.366]$ & 0.007 & $1.609[0.834-3.106]$ & 0.156 \\
Hemoglobin & $0.768[0.672-0.878]$ & $<0.001$ & $0.861[0.721-1.028]$ & 0.98 \\
CRP & $1.087[0.996-1.187]$ & 0.06 & $1.034[0.941-1.136]$ & 0.488 \\
Pre-procedural e-GFR & $1.014[0.999-1.03]$ & 0.06 & $1.022[1.002-1.043]$ & 0.033 \\
Contrast Media & $1.012[1.002-1.023]$ & 0.023 & $1.017[1.002-1.031]$ & 0.022
\end{tabular}

Abbreviations: PNI-prognostic nutritional index; DM-diabetes mellitus; CRP-C-reaktive protein; e-GFR-estimated glomerular filtration rate

CIN [13]. Moreover, recent studies have indicated a strong association between inflammatory markers and CIN [14]. Kaya et al. stated that the neutrophil-lymphocyte ratio (NLR) was an independent predictor of CIN in STEMI patients undergoing coronary intervention [15]. Sun et al. demonstrated that a higher platelet-lymphocyte ratio (PLR) was an independent risk factor for CIN development in patients with STEMI who underwent PCI [9]. Also, Yuan et al. evaluated the predictive value of relevant inflammatory factors on CIN development including; white blood cell count, NLR, CRP level, hs-CRP level, and endothelin-1 level on CIN development, in patients undergoing an emergency PCI and the results had shown that inflammatory factors were all associated with an increased risk of CIN development in patient group [14]. Additionally, recent studies have suggested that malnutrition, like inflammation, may play an important role in the development of CIN [16,17]. Chen and colleagues showed that malnutrition is associated with a twofold increased risk of CIN in patients with chronic artery disease undergoing coronary angiography [17]. Therefore, we thought that PNI, which contains information about both inflammation and nutritional status, may be useful in predicting CIN.

In the present study, the patients that developed CIN had significantly lower PNI scores than those that not developed CIN, and lower PLR was an independent risk factor for CIN development in patients with coronary artery disease undergoing PCI. We thought that the connection between PNI and CIN is not based on a cause-effect relationship. Both of these conditions are probably clinical manifestations of the same physiological impairments, particularly inflammation. As mentioned before, PNI is based on serum albumin levels and the lymphocyte count. Both serum albumin levels and lymphocyte count are related to inflammation. Therefore, a high frequency of CIN development in the low PNI score group can be an expected situation. Besides, the CRP level, a biomarker that reflects inflammation, was higher in the low PNI score group, which supports that idea.

PNI scores in chronic kidney disease patients were evaluated only in a few studies. Two of these studies were conducted in peritoneal dialysis patients $[18,19]$. Low PNI scores were associated with cardiovascular mortality, comorbidities, and impaired immuno-nutritional status in peritoneal dialysis patients. In another study, lower PNI scores were associated with decreased GFR in patients with acute heart failure [7]. Only one study explored the relationship between the PNI score and AKI [20]. Dolapoglu et al. investigated the predictive value of PNI regarding the development of AKI after elective coronary artery bypass grafting (CABG) surgery. They demonstrated that PNI was an independent predictor of AKI in patients undergoing on-pump CABG surgery [20]. In this study, we showed the relationship between low PNI scores and CIN.

The present study has several limitations. Firstly, this was a single-center, retrospective observational study. Second, the present study was conducted in high-risk group patients so the 
rate of CIN development was very high. We believe that PNI is a reliable indicator of CIN. However, it should be validated in the ordinary population. Third, inflammation-associated markers other than WBC counts and CRP were not analyzed or not compared with the PNI score.

\section{Conclusion}

This study demonstrated that PNI, a novel immunenutritional index, was an independent predictor of CIN development in patients undergoing CAG or PCI. Since PNI is simple and easy to be applied, it has the potential to become a convenient tool to stratify the risk of CIN development.

Disclosures: There is no conflict of interest for all authors.

\section{Acknowledgement: None}

Funding: None.

\section{References}

1. Akash Nair Sethi, Jatinder Kohli, Ami M, Patel and Michael R. Contrast induced nephropathy. In: Lerma EV, Sparks MA, Topf JM, editors. Nephrology Secrets. 4th ed. Philedelphia: Elsevier; 2019. p. 94-8. https://doi.org/10.1016/B978-0-323-47871-7.00022-8

2. McCullough PA, Wolyn R, Rocher LL, Levin RN, O'Neill WW. Acute renal failure after coronary intervention: incidence, risk factors, and relationship to mortality. Am J Med. 1997;103(5):368-375. https://doi.org/10.1016/s0002-9343(97)00150-2

3. Ali A, Bhan C, Malik MB, Ahmad MQ, Sami SA. The Prevention and Management of Contrast-induced Acute Kidney Injury: A Minireview of the Literature. Cureus. 2018;10(9):e3284. https://doi.org/10.7759/cureus.3284

4. Jeon HG, Choi DK, Sung HH, Jeong BC, Seo SI, Jeon SS, Choi HY, Lee HM. Preoperative Prognostic Nutritional Index is a Significant Predictor of Survival in Renal Cell Carcinoma Patients Undergoing Nephrectomy. Ann Surg Oncol. 2016;23(1):321-327. https://doi. org/10.1245/s10434-015-4614-0

5. Chan AW, Chan SL, Wong GL, Wong VW, Chong CC, Lai PB, Chan HL, To KF. Prognostic Nutritional Index (PNI) Predicts Tumor Recurrence of Very Early/Early Stage Hepatocellular Carcinoma After Surgical Resection. Ann Surg Oncol. 2015;22(13):4138-4148. https://doi.org/10.1245/s10434-015-4516-1

6. Chen QJ, Qu HJ, Li DZ, Li XM, Zhu JJ, Xiang Y, Li L, Ma YT, Yang YN. Prognostic nutritional index predicts clinical outcome in patients with acute ST-segment elevation myocardial infarction undergoing primary percutaneous coronary intervention. Sci Rep. 2017;7(1):3285. https://doi.org/10.1038/s41598-017-03364-X

7. Cheng YL, Sung SH, Cheng HM, Hsu PF, Guo CY, Yu WC, Chen CH. Prognostic Nutritional Index and the Risk of Mortality in Patients With Acute Heart Failure. J Am Heart Assoc. 2017;6(6):e004876. https://doi.org/10.1161/JAHA.116.004876

8. Mohri Y, Inoue Y, Tanaka K, Hiro J, Uchida K, Kusunoki M. Prognostic nutritional index predicts postoperative outcome in colorectal cancer. World J Surg. 2013;37(11):2688-2692. https://doi.org/10.1007/s00268-013-2156-9

9. Sun XP, Li J, Zhu WW, Li DB, Chen H, Li HW, Chen WM, Hua Q. Platelet to Lymphocyte Ratio Predicts Contrast-Induced Nephropathy in Patients With ST-Segment Elevation Myocardial Infarction Undergoing Primary Percutaneous Coronary Intervention. Angiology. 2018;69(1):71-78. https://doi.org/10.1177/0003319717707410

10. Stevens LA, Claybon MA, Schmid CH, Chen J, Horio M, Imai E, Nelson RG, Van Deventer M, Wang HY, Zuo L, Zhang YL, Levey AS. Evaluation of the Chronic Kidney Disease Epidemiology Collaboration equation for estimating the glomerular filtration rate in multiple ethnicities. Kidney Int. 2011;79(5):555-562. https://doi.org/10.1038/ki.2010.462

11. Onodera T, Goseki N, Kosaki G. [Prognostic nutritional index in gastrointestinal surgery of malnourished cancer patients]. Nihon Geka Gakkai Zasshi. 1984;85(9):1001-1005. Japanese. PMID: 6438478.

12. Hassan, M., Abdel Rehim, A. E., Khalil, M., Mahmoud Osman, Y. Nutritional assessment of cirrhotic patients with variable severity. Journal of Current Medical Research and Practice, 2019;4(2):144-151. https://doi.org/10.4103/JCMRP.JCMRP_14_18

13. Toso A, Leoncini M, Maioli M, Tropeano F, Di Vincenzo E, Villani S, Bellandi F. Relationship between inflammation and benefits of early high-dose rosuvastatin on contrast-induced nephropathy in patients with acute coronary syndrome: the pathophysiological link in the PRATO-ACS study (Protective Effect of Rosuvastatin and Antiplatelet Therapy on Contrast-Induced Nephropathy and Myocardial Damage in Patients With Acute Coronary Syndrome Undergoing Coronary Intervention). JACC Cardiovasc Interv. 2014;7(12):14211429. https://doi.org/10.1016/j.jcin.2014.06.023

14. Yuan Y, Qiu H, Hu X, Luo T, Gao X, Zhao X, Zhang J, Wu Y, Qiao S, Yang Y, Gao R. Predictive value of inflammatory factors on contrast-induced acute kidney injury in patients who underwent an emergency percutaneous coronary intervention. Clin Cardiol. 2017;40(9):719-725. https://doi.org/10.1002/clc.22722

15. Kaya A, Kaya Y, Topçu S, Günaydin ZY, Kurt M, Tanboğa IH, Kalkan K, Aksakal E. Neutrophil-to-lymphocyte ratio predicts contrastinduced nephropathy in patients undergoing primary percutaneous coronary intervention. Angiology. 2014;65(1):51-56. https://doi. org/10.1177/0003319713484789

16. Han M, Lee HW, Lee HC, Kim HJ, Seong EY, Song SH. Impact of nutritional index on contrast-associated acute kidney injury and mortality after percutaneous coronary intervention. Sci Rep. 2021;11(1):7123. https://doi.org/10.1038/s41598-021-86680-7

17. Chen L, Huang Z, Li W, He Y, Liang J, Lu J, Yang Y, Huang H, Lin Y, Lin R, Lin M, Liang Y, Hu Y, Ye J, Hu Y, Liu J, Liu Y, Fang Y, Chen K, Chen S. Malnutrition and the risk for contrast-induced acute kidney injury in patients with coronary artery disease. Int Urol Nephrol. 2021 Jun 25. https://doi.org/10.1007/s11255-021-02915-6

18. Kang SH, Cho KH, Park JW, Yoon KW, Do JY. Onodera's prognostic nutritional index as a risk factor for mortality in peritoneal dialysis patients. J Korean Med Sci. 2012;27(11):1354-1358. https://doi.org/10.3346/jkms.2012.27.11.1354

19. Peng F, Chen W, Zhou W, Li P, Niu H, Chen Y, Zhu Y, Long H. Low prognostic nutritional index associated with cardiovascular disease mortality in incident peritoneal dialysis patients. Int Urol Nephrol. 2017;49(6):1095-1101. https://doi.org/10.1007/s11255-017-1531-0

20. Dolapoglu A, Avci E, Kiris T, Bugra O. The predictive value of the prognostic nutritional index for postoperative acute kidney injury in patients undergoing on-pump coronary bypass surgery. J Cardiothorac Surg. 2019;14(1):74. https://doi.org/10.1186/s13019-0190898-7 